\title{
EL CENTRO DE EDUCACIÓN SECUNDARIA: ESPACIO DE CULTURA EN COLABORACIÓN. UNA PROPUESTA PERSONAL
}

\author{
Fermín Navaridas Nalda \\ Universidad de La Rioja
}

\begin{abstract}
RESUMEN. A través de este artículo se pretende reflexionar sobre la singularidad de los Centros de Educación Secundaria, especialmente los de Educación Secundaria Obligatoria (E.S.O.), y crear las bases para la mejora permanente de la institución.

Precisamente por el desafío que supone "la mejora continuada" de los centros educativos necesitará de la acción conjunta y cohesionada de toda la comunidad educativa (profesores, padres, alumnos, y otros agentes sociales del contexto educativo), dando lugar a espacios de reflexión y de elaboración de cultura.

Por último, una vez definidas las bases para crear la cultura y el clima de colaboración en el centro, se presenta a modo de conclusión una propuesta personal basada en los modelos didácticos instructivo-curriculares.
\end{abstract}

ABSTRACT. In this article a consideration about the singularity of the Educational Centers, particularly the Obligatory Secondary Educational Centers (E.S.O.), was made. Also some principles for the permanent improvement of the institution were created.

The continuity in the improvement process of the Educational Centers is a difficult target. Because of that is necessary the cohesive and jointly action of all the educational community (teachers, parents, students, and others social agents of the educational context) to create reflection and culture spaces.

Finally, once the collaboration atmosphere and the bases to create culture at the educational center were defined, a personal proposal based in the didactic and curriculum instuctive models was presented.

\section{Introducción}

El propósito del presente artículo es descubrir algunas claves educativas desde las que podamos crear Centros generadores de cultura en colaboración, críticos, transformadores e inmersos en un proceso de mejora continua.

En este sentido debemos comenzar reflexionando sobre la naturaleza y las bases para configurar la cultura y el clima de colaboración en los Centros de Educación Secundaria. Así, después de algunas conclusiones en términos generales, pasamos a describir una propuesta personal para la creación de la cultura de colaboración. Esta propuesta particular está basada en los modelos didácticos instructivos-curriculares y 
requiere de una serie de condiciones a través de las cuales podemos alcanzar nuestro objetivo en este trabajo.

Por último, como conclusión final del artículo, se trata de una reflexión que debe servirnos como fuente de hipótesis a partir de las cuales se pretenden futuras investigaciones que puedan ayudarnos a comprender mejor la singularidad de los Centros de Educación Secundaria.

\section{La Naturaleza de la cultura de colaboración}

Es indudable que el modelo curricular actual propuesto por la LOGSE (Ley Orgánica de Ordenación General del Sistema Educativo) concibe la PARTICIPACIÓN como un factor necesario y determinante en el buen funcionamiento de los centros.

El paso de un curriculum cerrado, determinado exclusivamente por el M.E.C. y las Comunidades Autónomas, a otro más abierto y flexible, en donde es responsabilidad de cada Centro educativo realizar, a partir del contexto socio-cultural de todos los miembros de la Comunidad educativa, su Planificación, constituye el núcleo de partida fundamental para crear una cultura de colaboración en las instituciones educativas.

Podríamos decir así que el PROYECTO DE CENTRO, en su sentido más amplio e integrador del Proyecto Educativo, Curricular y de Organización (como instrumentos comunes de gestión de la Comunidad educativa), es una buena oportunidad para que todos los miembros de la institución educativa trabajen en equipo y, por consiguiente, se obtenga como resultado una cultura propia de colaboración, aspecto importante para lograr una mejora cualitativa de la educación. Este trabajo en equipo, en palabras del profesor GENTO (1996: 150), debe partir "del compromiso en cada uno de los miembros del grupo para contribuir con su esfuerzo y en la medida que les corresponda a impulsar la consecución de los objetivos comunes. Pero, para que esta contribución individual o sectorial sea equitativa y con incidencia eficaz sobre los resultados, conviene que se lleve a cabo de acuerdo con una distribución previa de las responsabilidades a cubrir: de este modo, la contribución solidaria tendrá un efecto multiplicador sobre el esfuerzo de todos y cada uno de los miembros".

Es evidente que la discusión de los distintos miembros de la comunidad escolar en el PROCESO de diseño y desarrollo curricular, el análisis de su propia realidad de Centro, la negociación de principios, valores, objetivos didácticos, criterios curriculares que hayan tenido que consensuar a lo largo del proceso (a través de distintos órganos de gobierno y participación -Consejo escolar, Claustro de profesores, equipo directivo-, así como en otros órganos de coordinación -Departamentos didácticos, Comisión pedagógica, etc.- y asociaciones - APAs, de alumnos- ), supondrá un aprendizaje significativo y muy enriquecedor para todos (no solo para los alumnos, también para los profesores y los padres), conducente sin duda hacia la innovación y mejora continua. "Es ésta una de las ideas centrales de algunas de las propuestas actuales que abogan por una visión de los centros como organizaciones que aprenden" (ESCUDERO, 1997: 136).

Todo esto supone organizar el Centro de Educación Secundaria como un marco principal donde concurren las sucesivas interacciones de los miembros de la comunidad educativa, posibilitando de esta forma la colaboración de todos ellos. Así es 
muy importante que los centros posean una organización más flexible y autónoma de la que tenían hasta este momento con la Ley General de Educación de 1970. Deben de crearse espacios comunes para la discusión, hacer referencia continua a la práctica, experiencia y pensamiento de cada profesor, estructurar el centro en pequeños grupos de trabajo (p.ej.; equipos docentes, de padres de alumnos) que funcionen como estructuras operativas de toma de decisiones para hacer propuestas, fijar criterios y definir modelos, ofrecer recursos funcionales (todos aquellos elementos no materiales ni personales que posibiliten el funcionamiento del centro: tiempos, horarios, áreas, materias...) y favorecer el asesoramiento de agentes externos que agilicen y faciliten los procesos de discusión y toma de decisiones.

"La coincidencia es unánime cuando se plantea que la movilización de dichos recursos internos exige la colaboración de agentes externos -nos referimos genéricamente a ellos como asesores- con los necesarios conocimientos técnicos-pedagógicos, pero también con la sensibilidad necesaria para entender los centros como comunidades sociales con historia y cultura propias, en las que ningún proceso se puede implantar mecánicamente" (MARCELO GARCÍA, C; 1996: 27)

De todo lo dicho hasta aquí parece haber una conclusión unánime por todos: la riqueza pedagógica de un Proyecto de Centro (en el ámbito didáctico-organizativo), se dará preferentemente en aquellos centros que asuman de un modo coherente la colaboración.

Sin embargo, no siempre se está dispuesto a aceptar la responsabilidad que conlleva esta nueva línea de trabajo, optando por un modelo más centralizado y de toma de decisiones jerárquicas. Es decir, una forma de proceder como la vivida con el sistema educativo que nos precede (LGE de 1970), en donde la responsabilidad del Proyecto de Centro recae en la Administración y en quien ella delegue (generalmente lo hacía en los directores de los centros educativos). "Las consecuencias de una concepción tan racionalista no sólo afectan a lo organizativo sino que se derivan también a lo didáctico, donde el uso de programaciones rígidas, preestablecidas y minuciosamente ordenadas se justifican ampliamente. La actuación docente presta en este modelo una atención prioritaria a los conocimientos y olvida a menudo los factores intrínsecos que también inciden en lo educativo" (GAIRÍN SALLÁN, J.; 1997: 310). Se plantea, en este sentido, un modelo más preocupado por la cantidad que por la calidad (incoherente en una sociedad que se define como dinámica y que trata de educar para la libertad y la participación).

Parece, por tanto, conveniente desarrollar en las instituciones educativas una cultura de colaboración orientada a alcanzar la calidad total de sus proyectos. Objetivos como este, donde aumenta considerablemente la responsabilidad de todos los miembros de la comunidad escolar, conlleva un mayor nivel de formación (particularmente en los PROFESORES y, a ser posible, desde el propio Centro educativo) para responder adecuadamente a las nuevas exigencias.

Según MEDINA, A. (1995:143) "El sentido y valor de la formación del profesorado en el sistema radica en la permanente incidencia, que cada profesional de la educación tiene en la calidad del sistema educativo, y en la repercusión que una formación innovadora del profesorado tiene en la transformación de los procesos curriculares en el centro y aulas. Esta perspectiva de coprotagonismo con la innovación de la institu- 
ción educativa en su conjunto y su proyección en el resto del sistema educativo, sienta las bases de un nuevo modelo de formación del profesorado y de mejora global de la escuela".

Así, la LOGSE (Art..-56) señala que el centro escolar es responsable de la formación de su profesorado. Con la misma importancia en el Reglamento de I.E.S. se reconoce entre las competencias del Claustro de Profesores "participar en la planificación de la formación del profesorado del instituto y de elegir a sus representantes en el Centro de Profesores y Recursos".

Sin embargo, no solo es importante la formación de los profesores y alumnos del centro como organización que aprende desde una cultura de colaboración, sino que también lo es establecer programas de formación en el caso de los padres como parte responsable del proceso de enseñanza-aprendizaje de sus hijos. Es evidente que cuanto mayor sea la información y la formación de todos los miembros de la comunidad escolar, mejor será su clima relacional en la construcción de su Centro como Proyecto educativo de calidad.

"En resumidas cuentas, que la elaboración del Proyecto de Centro requiere de un conjunto de condiciones, clima, estructuras organizativas, liderazgo, asunción de compromisos y responsabilidades explícitas y concertadas. En ausencia de su adecuada orquestación, incluso nuestros mejores deseos pueden verse seriamente cercenados" (ESCUDERO, 1997:143).

\section{Bases para configurar la cultura y el clima de colaboración}

Establecer las bases para configurar la cultura y el clima de colaboración de un centro educativo significa, de inicio, partir del centro como un verdadero "grupo psicológico", que se organiza adecuadamente para elaborar y desarrollar de forma activa su propio proyecto educativo.

El Centro no sólo lo forman los docentes, sino también los padres, alumnos, profesores, responsables de administración y servicios, etc., son miembros de una comunidad comprometida a sacar un proyecto educativo adelante. De ahí que la cultura que se cree y el clima resultante es fundamental a lo largo del proceso de enseñaraprender.

Pero, ¿qué es la CULTURA y el CLIMA de -colaboración-?. Para el profesor MEDINA (1997:590-591) "la cultura es la configuración global de los estilos de reflexión, acción y modos de ser de los agentes del centro fruto de la tarea que llevan a cabo, las normas y estrategias que aplican, los roles y funciones y los objetivos que pretenden (...) El clima social es resultado del estilo de vida que configuran los miembros del centro y del aula."

Por tanto, cada comunidad educativa irá generando una cultura singular que le hará ser diferente de otras comunidades, con un clima social fruto de la organización, diseño y desarrollo de su proyecto de centro. Sin embargo, ¿hasta qué punto nuestros Centros de Educación Secundaria "viven" una cultura de colaboración?

Un conjunto de personas que están juntos en una sala, departamento, en el aula, etc..., pero que han de desempeñar su trabajo sin relacionarse con los demás, no es 
propiamente un "grupo" que asiente las bases para configurar la cultura y el clima de colaboración que nos preocupa. (Sin interacción no hay colaboración y sin colaboración difícilmente podemos llegar a un proceso de calidad. Hay determinadas actitudes que sólo podemos enseñarlas a través del ejemplo). En mi opinión, la cultura y el clima de colaboración empiezan a formar parte de un centro educativo cuando éste se convierte en un verdadero "grupo psicológico", en donde el respeto mutuo, la apertura, la conciencia de grupo... son aspectos normales que lo conforman.

Sin embargo, ¿Cuándo podríamos decir que el Centro educativo se ha convertido en un "grupo psicológico" capaz de generar esa cultura y clima de colaboración?. Autores como BALES (1935), HOMANS (1950), BONNER (1959), SHAW (1980), apuntan como elemento necesario para la existencia de un grupo la INTERACCIÓN FRECUEN$T E$ : “Un grupo es un conjunto de personas en interacción recíproca, y es este proceso de interacción lo que distingue un grupo de un agregado".

El concepto de INTERDEPENDENCIA amplía notablemente esta simple definición. KURT LEWIN es el máximo representante entre los autores que postulan algo más que el simple interaccionar para que el conjunto de individuos (p.ej., la comunidad educativa de un centro) se constituya en un grupo generador de esa cultura y, por tanto, de un clima de colaboración entre sus miembros que lo componen.

Si queremos puntualizar en qué consiste esa interdependencia de los miembros de un Centro educativo, donde se asume la colaboración como principio didáctico, tenemos que oir a TH. NEWCOMB cuando dice: " los miembros de un grupo son interdependientes cuando COMPARTEN NORMAS respecto de algo (normas que pueden ser formales e informales); y cuando DESEMPEÑAN ROLES en alguna forma complementarios (de nuevo estos roles pueden ser formales e informales)".

Una comunidad educativa que comparte normas y roles se puede llamar verdaderamente un grupo con estructura para la colaboración, donde un hecho que afecta a uno de los miembros ( alumnos, profesores, padres...) es probable que afecte a todos los demás.

El marco de relaciones es sin duda la norma más importante del centro y debe recibir un tratamiento específico. El conjunto de normas (siempre CRISTALIZADAS; es decir, formuladas y aceptadas por todos los miembros del centro) deben de procurar un marco de relaciones de cooperación, confianza, empatía y autonomía, que se traduzca en un clima social favorecedor del proceso enseñanza- aprendizaje. Estas son ideas importantes que se plasman en el trabajo de los autores MEDINA, A: y SEVILLANO, M.L. (1994) cuando estudian el clima del centro como "la síntesis del conjunto de relaciones que en él se van generando".

De hecho, no se produce un clima positivo en el Centro educativo cuando muchas personas trabajan en lo mismo (p.ej., desde los Departamentos didácticos, el claustro de profesores, etc.) y compiten entre sí, cuando evitan las responsabilidades que deben asumir y se pasan los asuntos de uno a otro, cuando defienden cotos cerrados, o cuando se fuerza una homogeneidad en las personas para adaptarse a la dirección.

Así podemos quizá llegar a formular una definición más o menos completa de lo que debe ser un Centro educativo como "grupo psicológico" creador de una cultura 
y un clima de colaboración; entendiendo, como nos diría ESCUDERO (1997: 99), que "un tipo de Centro como el que estamos sugeriendo -generador de una cultura y un clima de colaboración- habría de construirse por dentro como una institución no solo dedicada a la gestión del curriculum y la educación, esta opción correspondería a una perspectiva pura y simplemente burocrática. Por el contrario, aunque múltiples tareas y contenidos propios han de ser gestionados, una cuestión fundamental es la cultura (creencias, significados, valores, compromisos, responsabilidades, relaciones, etc.) de la que un centro se pertreche y esfuerce en construir y promover para hacer significativa y valiosa dicha gestión".

Por tanto, en mi opinión, la cultura y el clima del centro ha de construirse desde la FORMACIÓN de la Comunidad educativa como un verdadero grupo; es decir:

Como un conjunto de personas (profesores, alumnos, padres y otros agentes sociales implicados en el Proyecto de Centro) que establecen frecuente interacción entre ellas. Se definen a sí mismas como miembros (se sienten integrados en ese Proyecto común de Centro), comparten normas en materia de interés común. Constituyen un sistema de roles interdependientes (basados en el respeto y la comprensión mutua). Se identifican unos con otros y, sobre todo, con un mismo modelo ideal. Persiguen un objetivo común (reflejado previamente en su Proyecto educativo y asumido por los miembros como propio) u objetivos interdependientes; y tienen colectiva percepción de su unidad (conciencia de grupo) y de su diferencia con otros centros y partes del ambiente (su propia cultura de centro, en un contexto singular y con unas necesidades educativas concretas).

Sin duda alguna que la calidad de la enseñanza, la innovación curricular, la crítica constructiva desde los distintos espacios de reflexión ofrecidos por la organización del centro, la participación de toda la comunidad educativa en un Proyecto común, ... solo será posible promoviendo un clima de intercambio, de respeto, de seguridad y confianza, síntesis todo ello de una cultura de colaboración.

\section{Propuesta personal para la creación de "La Cultura de Colaboración" en centros de educación secundaria. Un modelo instructivo curricular para la innovación educativa}

La propuesta que a continuación se describe no ha de ser interpretada como una "receta mágica" capaz de crear en cualquier Centro de Educación Secundaria una cultura de colaboración y, ni mucho menos, resolver las distintas situaciones de "conflicto" naturales en el diseño y desarrollo del Proyecto de centro (en su sentido más global). Más bien debe ser cada centro quien, en función de su contexto y condiciones, haga su propuesta particular inspirada en algún/os modelo/s didácticos. Es necesario precisar que aunque en este trabajo nos hemos planteado la educación desde los modelos instructivos curriculares (tales como los propuestos por STENHOUSE, 1984; GIMENO, 1981 y BENEDITO, 1987) es importante no perder de vista otros modelos didácticos que pueden ser complementarios y muy enriquecedores en función del Proyecto Educativo de cada centro, las características de los alumnos, la organización, etc. Por tanto, tras el análisis y conocimiento del contexto y de las potencialidades de 
los modelos, debemos escoger y adecuar aquellos que mejor se ajusten a nuestra realidad educativa.

El diseño y desarrollo curricular por los Centros de Educación Secundaria, su traducción práctica y su seguimiento y evaluación, requieren de una serie de condiciones sin las cuales es muy difícil alcanzar el objetivo que nos proponemos: crear Centros de Educación Secundaria generadores de cultura en colaboración, críticos, transformadores e inmersos en un proceso de mejora continua.

Con este afán se realiza la siguiente propuesta:

\section{a) FORMAR EL CENTRO COMO "GRUPO" CON ENTIDAD PROPIA}

Los centros educativos deben ser realidades distintas de los miembros que lo componen. Se pretende crear una cultura de centro, donde la comunidad educativa tenga colectiva percepción de su unidad y de su diferencia con otros centros, donde se encuentre satisfacción profesional y personal por el hecho de pertenecer al mismo centro, se compartan normas en materia de interés común, persigan objetivos comunes e interdependientes. Es decir, el centro debe ser "algo más" que conjuntos de valores, ideas, conceptos, pensamientos, hábitos, etc., que existen en los miembros que forman parte de la comunidad escolar.

\section{b). ELABORAR EL CENTRO}

Una vez formado el centro educativo como un verdadero grupo (psicológicamente hablando), es conveniente la formación de sub-grupos o equipos de trabajo que asuman las nuevas responsabilidades en la confección y desarrollo de su Proyecto de Centro.

Aunque este propósito exija una mayor coordinación puede resultar muy beneficioso para promover el intercambio de ideas y experiencias relacionadas con su propia práctica educativa $y$, de esta forma, ir construyendo criterios y decisiones vertebradoras del centro y su educación.

Estas relaciones que se establecen en el diseño y desarrollo del Proyecto de Centro (Proyecto Educativo, Proyecto Curricular y Proyecto de Organización) van configurando un peculiar estilo de interacción o cultura de colaboración que, a su vez, deriva en un clima social muy positivo para 'la vida en el centro'.

De ser así el resultado es la cohesión de la comunidad educativa y, con ella, una mejora de la comunicación interna, así como un aumento del nivel de satisfacción de los miembros de esta comunidad educativa (profesores, alumnos, padres,...).

Por tanto, es importante contemplar organizaciones flexibles, espacios apropiados y tiempos suficientes que faciliten y potencien la interacción a la que nos estamos refiriendo en este apartado.

\section{c). CONTEXTUALIZAR EL CURRICULUM A LA REALIDAD ESCOLAR}

Es muy importante atender a las diferencias individuales y al contexto social, cultural y geográfico donde se aplica el proyecto. Para una contextualización del curriculum más precisa y efectiva, será necesaria la recopilación de datos concretos y 
actualizados del entorno externo e interno del propio centro en que se encuadra el alumno: familiar, cultural, social, laboral, académica, etc.

\section{d). LA FORMACIÓN DEL PROFESORADO COMO INNOVADOR DEL CENTRO}

Con la nueva Reforma educativa ha recaído sobre el profesor un papel cada vez más importante, un rol cada vez más amplio y complejo repleto de nuevas responsabilidades, fruto de las propuestas curriculares abiertas que en la actualidad elabora la Administración educativa. Las nuevas líneas de trabajo exigen que los profesores adapten y desarrollen los programas e intenciones educativas generales a las características y necesidades de los centros, consolidar la autonomía de sus centros, participar en las tareas de actualización permanente, innovar la metodología, etc.

Por tanto, es preciso potenciar desde el propio Centro educativo un nuevo modelo de formación permanente del profesorado que de respuesta a sus necesidades tanto personales como profesionales.

Esta formación permanente debe surgir a partir de la propia reflexión del profesor sobre su práctica docente y, a su vez, es muy importante que pueda compartirla e intercambiar ideas con el resto de compañeros. Este trabajo en colaboración será más enriquecedor y, sin duda, tendrá como consecuencia soluciones más creativas a los distintos problemas detectados durante el trabajo diario en el centro.

\section{e) INVESTIGACIÓN-ACCIÓN}

De esta forma el profesor se convierte en un investigador que es capaz de construir su propia teoría de la educación y, por tanto, de innovar el proceso de enseñanza-aprendizaje en colaboración con los demás profesionales de la enseñanza.

Según STENHOUSE (1.984) "es el docente con conocimiento de las características del alumno quien determina experiencias y selecciona los contenidos y primeras estrategias, para, después, por medio de la INVESTIGACIÓN-ACCIÓN resolver problemas que se plateen y adecuar mejor las estrategias, los medios y soportes de la organización escolar de conformidad con las necesidades contextuales" (cit. por JIMENEZ, B.; 1.989: 226).

\section{Conclusión}

Como conclusión final de este artículo podríamos afirmar que la cultura y el clima de colaboración en los Centros de Educación Secundaria, especialmente los de Educación Secundaria Obligatoria, son requisitos importantes y necesarios al objeto de conseguir lo establecido en la LOGSE como finalidad principal de la etapa:

"Transmitir a todos los alumnos los elementos básicos de la cultura, formarles para asumir sus deberes y ejercer sus derechos y prepararles para la incorporación a la vida activa o para acceder a la formación profesional específica de grado medio o al bachillerato " (Art.- 18).

Si los valores que difunden los profesores son contradictorios, si cada profesional de la enseñanza establece un nivel de exigencia individual y desproporcionado respecto al resto de compañeros, si se vive un clima de rivalidad y no existe en el Centro 
una cultura de colaboración, si no se asumen nuevas responsabilidades y la formación permanente del profesorado pasa a un segundo plano, si no se establecen espacios y tiempos para el intercambio de experiencias por los distinto miembros de la comunidad educativa, la Institución Educativa se convierte en un "elemento deformador", quedando muy lejos la finalidad de la etapa a la que nos hemos referido con anterioridad.

\section{Referencias Bibliográficas}

CARDONA ANDÚJAR, J. (COORD) (1994). Metodología Innovadora de Evaluación de Centros Educativos. Madrid. Sanz y Torres

ESCUDERO, J.M. (COORD.) y OTROS (1997). Diseño y desarrollo del currículum en Educación Secundaria. Barcelona, Hortisori,

GAIRÍN SALLÁN, J.: El contexto escolar. En MEDINA, A. y SEVILLANO, M.L. (COORDS.) (1997). El currículum: Fundamentación, diseño, desarrollo y evaluación. Madrid. UNED

GENTO PALACIOS, S. (1996). Instituciones educativas para la calidad total. Madrid, La Muralla.

GIMENO, J. Y PÉREZ GÓMEZ, A. (1983). La enseñanza: su teoría y su práctica. Madrid. Akal.

JIMÉNEZ, B., GONZÁLEZ, A.P. y FERRERES, V.(1989). Modelos didácticos para la innovación educativa. Barcelona, PPU.

MARCELO, C. (DTOR.) (1996). Innovación educativa, asesoramiento y desarrollo profesional. Madrid. CIDE.

MARÍN IBAÑEZ, R. : Los contenidos actitudinales en el marco de La Reforma. En SEVILLANO, M.L. (COOR.) (1995). Estrategias de enseñanza y aprendizaje con medios y tecnología. Madrid. Centro de Estudios Ramón Areces.

MARTÍN MORENO, Q. (COORD.) (1989). Organizaciones educativas. Madrid. UNED.

MEDINA, A. y SEVILLANO, M.L. (1994). El clima relacional del centro. Vídeo. Madrid, UNED.

MEDINA, A (1995). Formación del profesorado e innovación curricular. Bordón, 47, (2), 143-160.

MEDINA, A. y SEVILLANO, M.L. (COORDS.) (1997). El currículum: Fundamentación, diseño, desarrollo y evaluación. Madrid. UNED.

SANTOS GUERRA, M.A. (1987). Organización escolar e investigación educativa.. Investigación en la escuela, nํㅡ, pp. 3-13.

SHAW, M.E. (1980). Dinámica de grupos . Psicología de la conducta de los pequeños grupos. Barcelona, Herder. 


\section{Fundamentos Legales:}

Ley Orgánica de Ordenación General del Sistema Educativo 1/1990, de 3 de Octubre.

Ley Orgánica 9/1995, de 20de noviembre, de la Participación, la Evaluación y el Gobierno de los centros docentes (B.O.E. del 21-11-95)

OO.MM. de 12 de noviembre de 1992 (BOE del 30), sobre la evaluación en Educación Primaria y Secundaria.

REAL DECRETO 1345/91 de 6 de septiembre (B.O.E . 13-09-091) por el que se establece el currículo de la Educación Secundaria Obligatoria. Modificado en el REAL DECRETO 1390/1995 de 4 de agosto (BOE 19-09-95).

REAL DECRETO 1007/91 de 14 de junio (B.O.E . 26-06-091) por el que se establecen las enseñanzas mínimas correspondientes a la Educación Secundaria Obligatoria. Corrección de errores en el BOE 01-08-91. 